\title{
Revista Colombiana de

\section{Cierre de ruptura del seno de Valsalva no coronariano con dispositivo de Amplatzer asociado a implante de válvula aórtica transcateterismo}

\section{Celin Malkun Paz*, Alfonso Muñoz Velásquez, Oscar Isaac Cure, Jorge Escobar Modesto y Carlos Rebolledo Maldonado}

\author{
Clínica Iberoamérica, Barranquilla, Colombia
}

Recibido el 6 de septiembre de 2017; aceptado el 19 de enero de 2018

Disponible en Internet el 18 de abril de 2018

\section{PALABRAS CLAVE \\ Cierre percutáneo; Seno de Valsalva; Estenosis aórtica; Implante percutáneo válvula aórtica}

\begin{abstract}
Resumen
Objetivo: Reportar un caso de implante de cierre de ruptura del seno de Valsalva e implante de válvula aórtica transcateterismo.

Material y métodos: Paciente femenina de 82 años, con diagnóstico de estenosis valvular aórtica severa asociada con ruptura del seno de Valsalva no coronariano a la aurícula derecha e hipertensión arterial pulmonar severa, en quien, por sus comorbilidades, se decidió efectuar cierre percutáneo de la ruptura del seno de Valsalva con dispositivo de Amplatzer y posterior implante de válvula aórtica por cateterismo.

Resultados: Se realiza procedimiento percutáneo de implante del dispositivo de cierre Amplatzer VSD Occluder de $8 \mathrm{~mm}$ sellando la ruptura del seno de Valsalva no coronariano. En segundo tiempo se realiza procedimiento de Implante de válvula aórtica transcateterismo vía femoral derecha. Se corrobora su adecuada implantación mediante angiografía y ecocardiograma transesofágico. Se considera exitoso el procedimiento ya que se implantaron ambos dispositivos en forma rápida, adecuada y sin complicaciones.

Conclusiones: Se reporta un caso de cierre de ruptura de seno coronario con dispositivo Amplatzer e implante de prótesis aórtica transcateterismo.

(c) 2018 Sociedad Colombiana de Cardiología y Cirugía Cardiovascular. Publicado por Elsevier España, S.L.U. Este es un artículo Open Access bajo la licencia CC BY-NC-ND (http:// creativecommons.org/licenses/by-nc-nd/4.0/).
\end{abstract}

\footnotetext{
* Autor para correspondencia.

Correo electrónico: cmalkun@yahoo.com (C. Malkun Paz).
} 


\section{KEYWORDS}

Percutaneous closure; Sinus of Valsalva; Aortic stenosis; Percutaneous implant of aortic valve

\section{Amplatzer device closure of a ruptured sinus of Valsalva aneurysm associated with a transcatheter aortic valve implant}

\begin{abstract}
Objective: To report the case of an implant for the closure of a Valsalva sinus rupture and implantation of a transcatheter aortic valve.

Material and methods: An 82 year-old female patient, with a diagnosis of severe aortic valve stenosis associated with a non-coronary rupture of the sinus of Valsalva, at the right atrium, as well as severe pulmonary arterial hypertension. Due to her comorbidities, it was decided to perform a percutaneous closure of the sinus of Valsalva rupture with an Amplatzer device, and a subsequent aortic valve implantation by catheterisation.

Results: A percutaneous procedure was performed by implanting an $8 \mathrm{mmAmplatzer} \mathrm{VSD} \mathrm{Occlu-}$ der device, sealing the non-coronary rupture of the sinus of Valsalva. In a second time, an aortic valve was implanted using transcatheterisation via the right femoral artery. It was checked that it was appropriately implanted using angiography and a transoesophageal echocardiogram. The procedure was considered successful, since both devices could be implanted rapidly, adequately, and with no complications.

Conclusions: The case is reported of the closure of the sinus of Valsalva rupture with an Amplatzer device and the transcatheterisation implantation of an aortic valve.

(C) 2018 Sociedad Colombiana de Cardiología y Cirugía Cardiovascular. Published by Elsevier España, S.L.U. This is an open access article under the CC BY-NC-ND license (http:// creativecommons.org/licenses/by-nc-nd/4.0/).
\end{abstract}

Las fístulas y los aneurismas del seno de Valsalva rotos son raras anomalías del corazón. En estudios ecocardiográficos se encontró una prevalencia del $0,02 \%{ }^{1}$. Pueden ser congénitas o adquiridas, habitualmente secundarias a procesos degenerativos, infecciosos o quirúrgicos ${ }^{2}$. En general, tienen aspecto de túnel y en algunos casos pueden ser rupturas simples del seno a las cavidades en forma directa, por lo que se da el nombre de "ruptura del seno de Valsalva".

La causa más frecuente de las fístulas del seno de Valsalva es la perforación de un aneurisma congénito de un seno de Valsalva, si bien también existen otras etiologías, como las infecciosas (endocarditis, aortitis luética), traumáticas o iatrogénicas ${ }^{1}$ y la disección aórtica. Existen pocos reportes de rupturas aisladas sin presencia de aneurismas del seno de Valsalva ${ }^{3}$.

Los aneurismas del seno de Valsalva inicialmente fueron descritos por Hope en 1839 y un año más tarde se reportó por primera vez la ruptura de un aneurisma del seno de Valsalva a cavidades cardiacas ${ }^{4}$. Pueden ser de origen congénito (Marfan, Ehlers-Danlos y Behçet) e infeccioso (sífilis, tuberculosis o endocarditis) o deberse a enfermedades degenerativas e inflamatorias (aterosclerosis, necrosis cística de la media). Se han descrito casos secundarios a traumas por desaceleración.

Fueron clasificados en 1962 por Sakakibara y Konno ${ }^{4}$. Posteriores modificaciones ${ }^{5}$ han esclarecido su fisiopatología y lugar de ruptura; no obstante, se han reportado diferentes casos de ruptura aislada no asociada a aneurismas ${ }^{6-12}$.

Comúnmente se dan en el seno de Valsalva coronariano derecho $(65 \%-85 \%)$, en el seno de Valsalva no coronariano $(10 \%-30 \%)$ y en el coronariano izquierdo $(<5 \%)$. En el seno de Valsalva coronariano derecho la ruptura ocurre en el ventrículo derecho, mientras que la ruptura del seno de Valsalva no coronariano se da en la aurícula derecha. Se estima que la ruptura de aneurismas del seno de Valsalva ocurre en el $40 \%$ y $76 \%$ de los pacientes ${ }^{6-15}$.

\section{Caso}

Paciente de sexo femenino, de 82 años de edad, remitida en agosto de 2016 al servicio de Hemodinamia de la Clínica Iberoamérica, para implante de válvula aórtica transcatéter (TAVI) por diagnóstico de doble lesión aórtica con estenosis predominante y alto riesgo para reemplazo valvular aórtico por cirugía, ya que padecía hipertensión arterial pulmonar severa clasificada como tipo primario, además de antecedentes de hipertensión arterial sistémica e insuficiencia renal en fase no dialítica.

Al examen físico de ingreso presentó presión arterial de $190 / 90 \mathrm{~mm} \mathrm{Hg}$, frecuencia cardiaca de $70 \mathrm{lpm}$, peso $64 \mathrm{~kg}$ y talla 1,60 m. El electrocardiograma mostró ritmo sinusal con bloqueo aurículo-ventricular de primer grado e hipertrofia ventricular izquierda. Previamente le había realizado cateterismo cardiaco que evidenció válvula aórtica calcificada con apertura disminuida e insuficiencia leve a moderada. Las arterias coronarias no mostraban lesiones ateroscleróticas significativas. Fue presentada en junta médico quirúrgica de la institución de origen y se determinó que debido a sus comorbilidades, era de alto riesgo para cirugía de reemplazo valvular aórtico con cálculo de riesgo por Euroscore aditivo de 14 y logístico de $50 \%$, y riesgo según el índice de la Sociedad de cirugía torácica (STS) de 5,5, motivo por el cual fue remitida para implante percutáneo de válvula aórtica.

El 12 de agosto de 2016 se hizo ecocardiograma (fig. 1A) que mostró hipertrofia parietal concéntrica importante del ventrículo izquierdo con estenosis aórtica calcificada. Mediante Doppler color se detectó, además, cortocircuito 

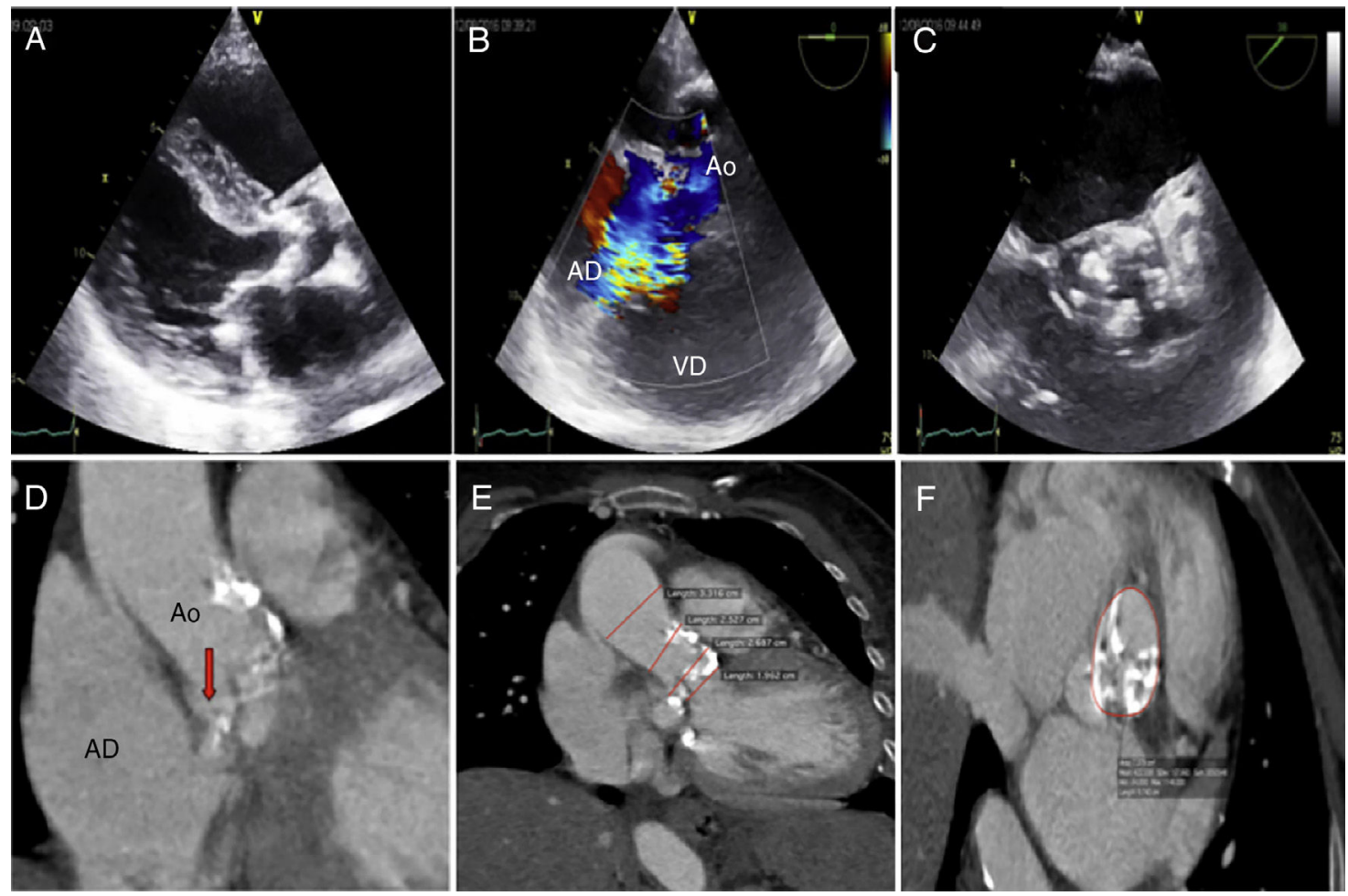

Figura 1 (A) Ecocardiograma bidimensional en el que se observa válvula aórtica calcificada, hipertrofia ventricular concéntrica y calcificación de la valva posterior de la mitral. (B) Ecocardiograma color que muestra cortocircuito entre el seno de Valsalva no coronariano a la aurícula derecha. (C) Ecocardiograma eje corto que muestra deformidad del seno de Valsalva y calcificación aórtica. (D, E, F) Angio-tomografia de corazón y aorta en la que se corrobora ruptura del seno no coronariano a la aurícula derecha y diferentes medidas necesarias para implante de la prótesis.

desde el seno de Valsalva no coronariano a la aurícula derecha por trayecto fistuloso de $4 \mathrm{~mm}$ de diámetro (fig. 1B). El Doppler transvalvular aórtico encontró gradiente pico de $91 \mathrm{~mm} \mathrm{Hg}$ y gradiente medio de $49 \mathrm{~mm} \mathrm{Hg}$. El ecocardiograma también demostró hipertensión arterial pulmonar con cálculo de la presión sistólica de la arteria pulmonar de $70 \mathrm{~mm} \mathrm{Hg}$. Ante estos hallazgos se determinó hipertensión arterial pulmonar secundaria a cortocircuito de izquierda a derecha por ruptura del seno de Valsalva no coronariano a la aurícula derecha en forma asociada a la estenosis aórtica severa.

Se presentó en junta médico-quirúrgica de nuestra institución y se aprobó el cierre percutáneo de la ruptura del seno de Valsalva no coronariano a la aurícula derecha. Se solicitó angiotomografía de corazón y de aorta toraco-abdominal como protocolo de implante de válvula aórtica por cateterismo, en el que se corroboró la ruptura del seno de Valsalva no coronario a la aurícula derecha (fig. 1D). Se hicieron las mediciones de la válvula aórtica como protocolo para la elección del tipo de prótesis a utilizar.

El día 28 de agosto de 2016 se programó cierre de fistula con dispositivo de Amplatzer Muscular VSD Occluder de $8 \mathrm{~mm}$ vía radial derecha, el cual se llevó a cabo bajo anestesia general y mediante la guía de ecocardiograma transesofágico. Se canalizó arteria radial derecha y se colocó introductor arterial 6 French; se pasó catéter multipropósito 5 French en la raíz de aorta. Se anticoaguló con
6.000 unidades de heparina y se instauró antibiótico-terapia profiláctica con dos gramos de cefazolina intravenosa. Mediante visualización fluoroscópica se introdujo guía hidrofílica recta de 0,014"' de $260 \mathrm{~cm}$; se logró pasar a la aurícula derecha a través de la fistula (fig. 2 A). Mediante técnica de Seldinger vía femoral derecha se puncionó la vena femoral derecha y se pasó un sistema de captura con guía canastilla Snare montada sobre catéter de 6 French y se capturó la guía hidrofílica en la aurícula derecha (fig. 2 B) extrayéndola por el introductor ubicado en la vena femoral derecha y a través del extremo de esta guía, se pasó catéter multipropósito el cual se dejó colocado en la aorta ascendente. Se procedió a retirar la guía hidrofílica de intercambio y se dejó una guía de alto soporte Amplatz 0.035'" pulgadas de $260 \mathrm{~cm}$. A través de esta guía se retiró el catéter multipropósito y pasó el sistema de liberación del dispositivo de cierre tipo Amplatzer antes descrito, se liberó el primer disco y se retrajo hasta dejarlo asegurado en el seno de Valsalva de la aorta para la liberación del disco distal en la aurícula derecha. A través de ecocardiograma transesofágico y angiografía se documentó el cierre de la ruptura del seno de Valsalva por lo que se procedió a liberar el dispositivo. Mediante ecocardiografía se evidenció disminución considerable de la presión sistólica de la arteria pulmonar de 70 a $50 \mathrm{~mm} \mathrm{Hg}$. No hubo complicaciones (fig. 3).

En segundo tiempo, al mes del cierre de la ruptura del seno de Valsalva, se programó para intervención percutánea de la válvula aórtica mediante dispositivo autoexpandible 


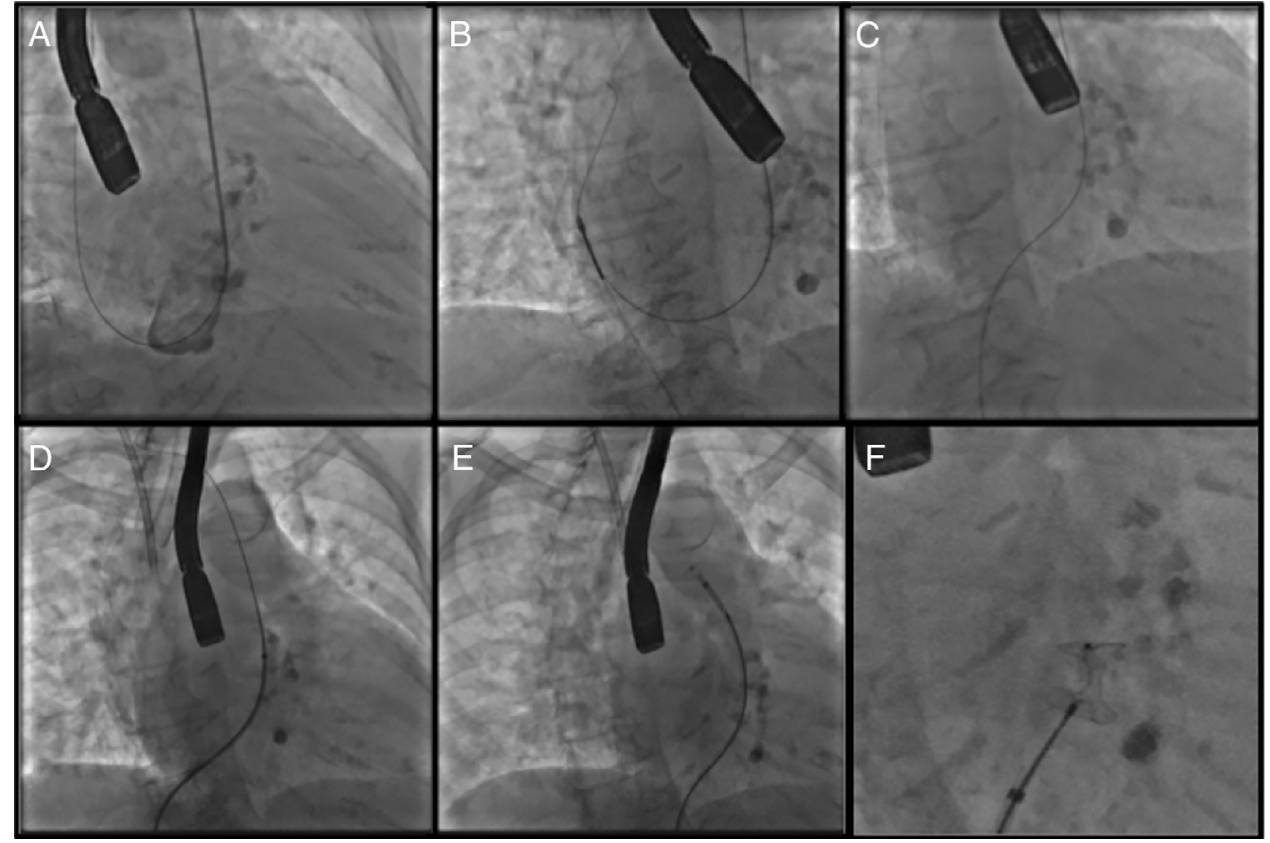

Figura 2 Procedimiento intervencionista del cierre de la ruptura del seno de Valsalva. (A) Paso de la guía vía arteria radia derecha a la aurícula derecha. (B) Se atrapa la guía con el Snare y se extrae por vía vena femoral derecha. (C) Se crea un asa con la guía entre arteria radial y vena femoral (D). Vía femoral izquierda se pasa catéter con el sistema de liberación del dispositivo de cierre. $(E, F)$ Colocación y liberación del dispositivo cerrando la ruptura.

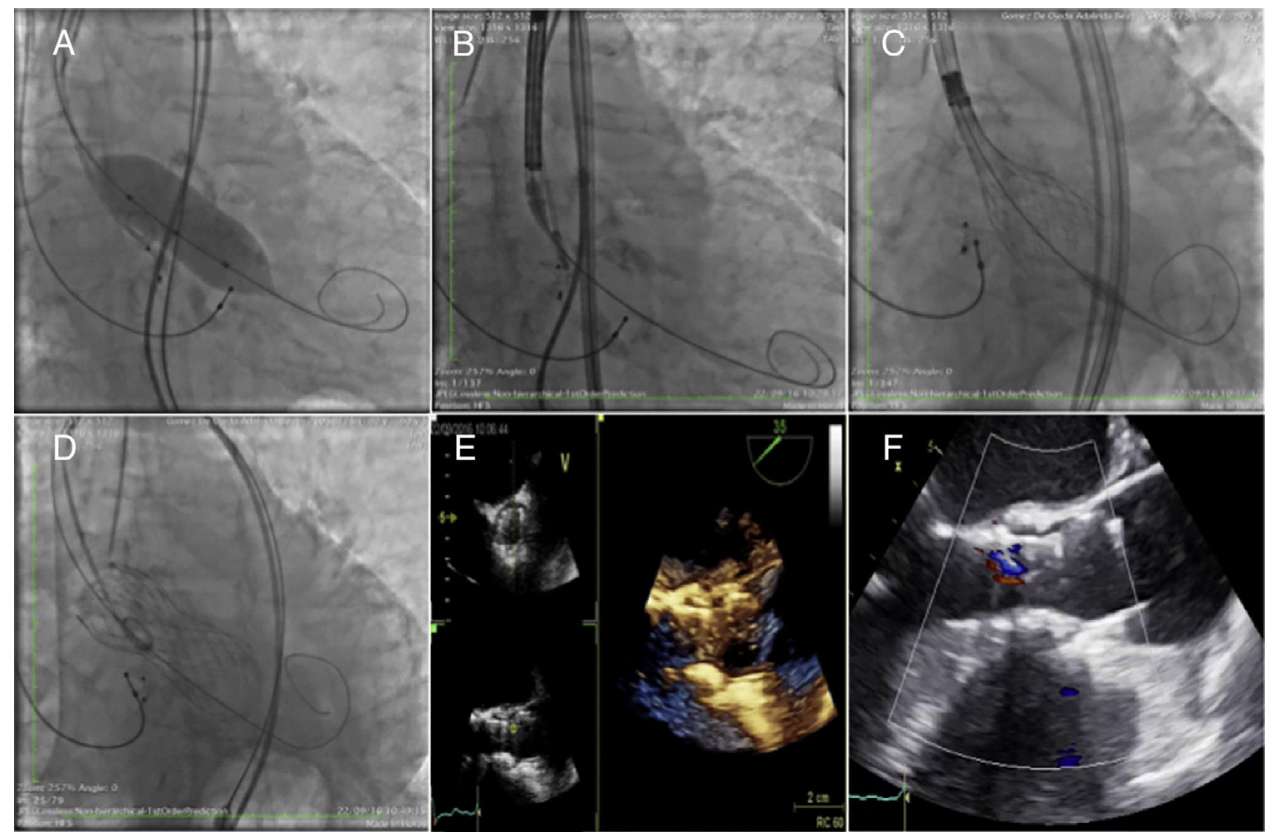

Figura 3 Procedimiento de implante de válvula aórtica transcateterismo (A). Valvuloplastia previa (B, C y D). Colocación y liberación de la válvula auto-expandible Corevalve ${ }^{\oplus}$. $(E, F)$ Imágenes del ecocardiograma intra-procedimiento en el que se corrobora la colocación adecuada y el funcionamiento de la prótesis aórtica.

Corevalve $^{\circledR}$ de Medtronic número 26 , cuyo implante se hizo vía femoral derecha mediante protocolo establecido. Este fue exitoso, no detectó gradiente residual y sí insuficiencia aórtica leve. No se presentaron complicaciones y se le dio de alta de la clínica al cuarto día del procedimiento de implante de la prótesis aórtica.

\section{Discusión}

Usualmente los aneurismas del seno de Valsalva rotos se reparan mediante cirugía. Esta reparación puede realizarse a través de la cámara de origen (aorta) o de la cámara de penetración o a través de $\operatorname{ambas}^{6}$. La técnica de elección 
es el cierre primario, el reemplazo de la raíz aórtica o el cierre con parche. En 1994, Cullen et al. ${ }^{13}$ reportaron el primer caso de cierre de una ruptura del seno de Valsalva en forma percutánea por cateterismo mediante sombrilla de Rashkind, la cual se usaba para cierre de ductus arterioso. Desde entonces se han publicado casos aislados y series de $\operatorname{casos}^{1,2,14,15}$ en los que se han tratado en forma percutánea el cierre mediante dispositivos con buenos resultados y bajos niveles de complicaciones. La necesidad de corregir las rupturas se basa en los síntomas del paciente, la alteración hemodinámica y la cámara en la cual se rompa el aneurisma. Los aneurismas del seno de Valsalva no rotos que producen arritmias malignas, infección, obstrucción de arterias coronarias o de tractos de salida ventricula condires, tienen indicación de cierre ${ }^{6}$.

Aunque la asociación entre aneurisma del seno de Valsalva congénito y estenosis aórtica se reporta en un $6 \%$ de todos los casos de aneurisma de seno del Valsalva ${ }^{13}$, es una condición infrecuente.

Dadas las comorbilidades de la paciente del caso y el riesgo quirúrgico que presentaba, se optó por el cierre percutáneo mediante dispositivo de Amplatzer e implante percutáneo de válvula aórtica transcatéter con prótesis auto-expandible. Ambos procedimientos se hicieron por separado ya que se tuvo en cuenta la recomendación de la casa matriz de la Corevalve ${ }^{\circledR}$, que sugirió que el implante de la misma fuera en un segundo tiempo. Hoy se sabe que no existe una contraindicación para realizar los dos procedimientos de manera simultánea, ya que se reducen, en teoría, a complicaciones de dos procedimientos por separado. En este caso, el cierre de la ruptura del seno de Valsalva se realizó mediante dispositivo de cierre de comunicación interventricular muscular ya que la morfología y el tamaño de la ruptura así lo ameritaban. No existen dispositivos específicos para el cierre de estos defectos, de modo que han utilizado diferentes tipos de dispositivos de Amplatzer para cierre de foramen ovale permeable y ductos, así como distintos dispositivos plug vasculares. La elección del dispositivo se basará en el tamaño del orificio de ruptura, la forma del mismo y la cavidad en la que se rompe, evaluando la funcionalidad de la válvula sin afectar estructuras vecinas.

\section{Conclusiones}

Se presenta el caso de una paciente con ruptura del seno de Valsalva y estenosis aórtica severa las cuales fueron resueltas de forma percutánea con éxito. La ruptura de aneurisma del seno de Valsalva puede ser corregida mediante implante de dispositivo de Amplatzer. Es un procedimiento seguro, que conlleva bajas complicaciones y es una alternativa eficaz frente a la cirugía. El cierre de fístulas del seno coronario puede hacerse de manera concomitante con implante de TAVI.

\section{Conflicto de intereses}

Ninguno.

\section{Agradecimientos}

A todos los médicos que colaboraron en el procedimiento y al personal paramédico y administrativo de la Clínica Iberoamérica por el apoyo prestado para la realización de este caso.

\section{Bibliografía}

1. Isturiz SDAC, Chacón M, et al. Fístula del seno de Valsalva derecho drenando a ventrículo derecho: resolución percutánea complicada exitosa. Avances Cardiol. 2014;34:328-30.

2. Cavellucci SM, Trandafilov D, et al. Fístula aorta-atrio derecho, simulando CIV con cierre percutáneo por prótesis CERA. Rev Bras Ecocardiogr Imagem Cardiovasc. 2013;26:125-8.

3. De la Fuente-Mancera JCB-BrE, Meave-González A. Rotura idiopática del seno derecho de Valsalva. Reporte de caso. Anales de Radiología México. 2014;13:128-31.

4. Sakakibara SKS. Congenital aneurysm of the sinus of Valsalva: anatomy and classification. Am Heart J. 1962;63:405-24.

5. Xin-Jin LXL, Bo P, Hong-Wei G, Wei W, Shou-Jun L, ShengShou H. Modified Sakakibara classification system for ruptured sinus of Valsalva aneurysm. J Thorac Cardiovasc Surg. 2013;146: 874-8.

6. Galicia-Tornell MMMn-SsB, Mercado-Astorga O, EspinozaAnguiano S, Martínez-Martínez M, Villalpando-Mendoza E. Aneurisma del seno de Valsalva roto. Informe de casos y revisión de la literatura. Cir Ciruj. 2009;77:473-7.

7. Choudhry LK, Rao VM, Gnanamuthu BR, Agrawal V, Shankar $\mathrm{R}$, Prasath R. Embolization of the device to the left pulmonary artery after the interventional closure of ruptured sinus of Valsalva aneurysm. The Korean journal of thoracic and cardiovascular surgery. 2015;48:202-5.

8. Abdelsalam MBW, Pawlush D, Mumtaz M, Goldman J. Noncoronary sinus of Valsalva aneurysm rupture into right atrium. Images in Cardiovascular Medicine. 2013;40:493-4.

9. Caon LB, Hannon DAM. Noncoronary sinus of Valsalva rupture into the right atrium with a coexisting perimembranous ventricular septal defect. World J Clin Cases. 2013;1: $146-8$.

10. John ESBJ, Ledzian B, Steward H, Moro R, Bittner HB. A rare case of sinus of Valsalva-right atrial fistula secondary to an abscess perforation from underlying aortic valve endocarditis. J Cardiothorac Surg. 2014;9.

11. Morais H, Martins T. Right Sinus of Valsalva Aneurysm with Rupture into the Interventricular Septum and into the Left Ventricle. Heart views: the official journal of the Gulf Heart Association. 2015;16:114-5.

12. Sanna GD, Talanas G, Denurra C, Ferrandu P, Bullitta L, Terrosu P. An unusual cause of acute chest pain: rupture of the noncoronary sinus of Valsalva into the right atrium. The Am J Emerg Med. 2016.

13. Cullen S, Somerville J, Redington A. Transcatheter closure of a ruptured aneurysm of the sinus of Valsalva. $\mathrm{Br}$ Heart $\mathrm{J}$. 1994;71:479-80.

14. Zhen-fei Fang, Yi-yuan Huang, Liang Tang, et al. Long-term outcomes of transcatheter closure of ruptured sinus Valsalva aneurysms using patent ductus arteriosus occluders. Circ J. 2014;78:2197-202.

15. Szkutnik M, Kusa J, Glowacki J, Fiszer R, Bialkowski J. Transcatheter closure of ruptured sinus Valsalva aneurysms with Amplatzer Occluder. Rev Esp Cardiol. 2009;62: 1317-21. 